\title{
VISUAL STUDIES ON RICE BRAN EXTRUSION
}

\author{
Wenhua Zhu ${ }^{1,}$, Huiyuan $\mathrm{Yao}^{2}$ \\ ${ }^{I}$ CIMS \& Robot Center of Shanghai University, Shanghai University, China; Email: \\ toney_wh_zhu@staff.shu.edu.cn. ${ }^{2}$ Food School, Southern Yantze University, China.
}

Abstract: This paper presents to make use of visualization technique to study the process of extrusion and extrusion assembly, the visual phenomena indicate that material transportation underlying the extrusion course is a combination of two transportations, one is positive displacement pumping transportation in the intermeshing region, and the other is partially filled transportation in the translation region. Virtual assembly technique is used to design the extrusion assembly, and interference is checked, and animations are created to demonstrate assembly sequence and path. This product has been designed successfully by computer in virtual environment.

Key words: Visualization technique, Virtual assembly, Rice bran, Extrusion.

\section{INTRODUCTION}

Nowadays visualization technique is a valid and useful mean to research the complicated problem of engineering that have not been reached and researched intensively. Owing to the complexity of process, extrude process is called as "Black Box" for many years, as is shown in the fig. $1^{[1]}$, we can see clearly that process equipment is a black box between the input and output. Visualization technique is adopted to research the order of rice bran extrusion, so that to provide the academic basis that designs the rice bran extruder and establishes theory model of rice bran extrusion, hence it will be made the theory model match the fact better.

This project is supported by the $10^{\text {th }}$ Five-year Plan Key Technologies R\&D Program of China under the grant No.2001BA501A03.

Please use the following format when citing this chapter:

Zhu, Wenhua, Yao, Huiyuan, 2006, in International Federation for Information

Processing (IFIP), Volume 207, Knowledge Enterprise: Intelligent Strategies In Product Design, Manufacturing, and Management, eds. K. Wang, Kovacs G., Wozny M., Fang M., (Boston: Springer), pp. 966-971. 
Input Extruder Output

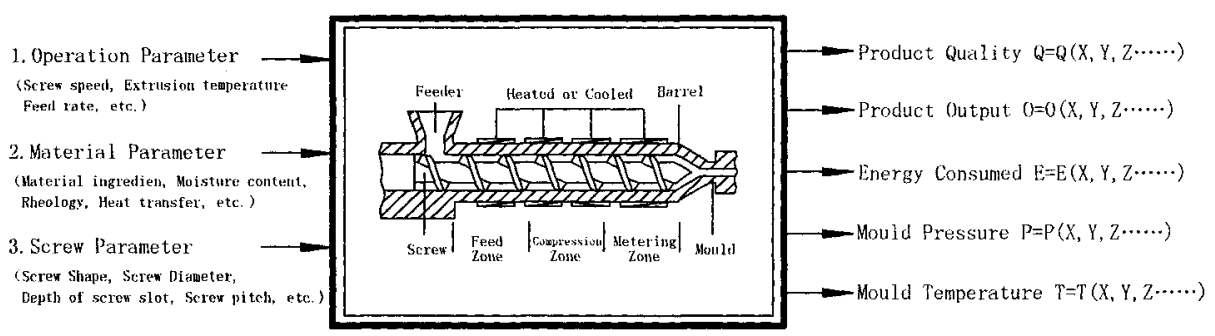

Figure 1. Black box model of food extruder

Rice bran is a sort of nutritious and functional food material, In order to develop rice bran resources, the first thing is how to stabilize rice bran. For the stabilization research of rice bran, there are many different approaches, J.R. Loeb etc. ${ }^{[2]}$ used refrigerating method to handle the rice bran, $M$. Williams and the S. Baer ${ }^{[3]}$ first time adopted extrusion to stabilize rice bran in 1965 , J.V. Prabhakar etc. ${ }^{[4]}$ stabilized rice bran with the chemistry method, J. Tao etc. ${ }^{[5]}$ utilized microwave to stabilize rice bran, Fernando etc. ${ }^{[6]}$ stabilized rice bran in the fluidized bed. It was latest reported by American RiceX company ${ }^{[7]}$, extrusion with high temperature and high pressure and high shearing is an economical and effective method, also become unique engineering method available. All these above researches do not mentioned visualization method, and this paper will study rice bran extrusion with visualization technique.

\section{VISUAL PHENOMENA AND ANALYSIS OF RICE BRAN EXTRUSION ${ }^{[8]}$}

\subsection{Positive displacement pumping transportation in the intermeshing region}

When rice bran is added to the barrel from feeder, one part of materials is piled up in the intermeshing region and transported forward along the length direction of screw, and another part of materials is overflowed to the under intermeshing region and also transported forward. This phenomenon is called positive displacement pumping transportation in the intermeshing region, fig.2(a) is the illustration and fig.2(b) is the visual picture 

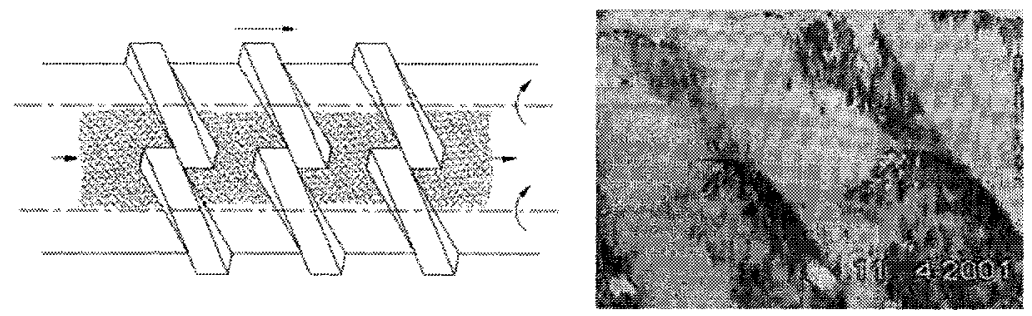

(a) Illustration of positive displacement. (b) Visual picture of positive displacement.

Figure 2. Positive displacement pumping transportation in the intermeshing region

\subsection{Partially filled transportation in the translation region}

When rice bran is added to the barrel from feeder, the other part of materials is transported in the side screw slot, rice bran entered side slot is moved upward into the upper intermeshing region by fiction drag, thus the consecutive movement of rice bran is formed the " $\infty$ " shape, but the screw slot isn't fully filled rice bran. This phenomenon is called partially filled transportation in the translation region, fig.3(a) is the illustration, fig.3(b) is the visual picture.

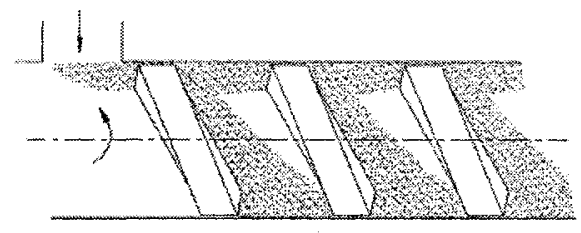

(a) Illustration

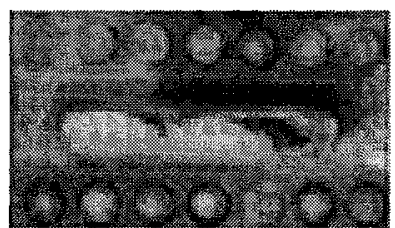

(b) Visual picture

Figure 3. Partially filled transportation in the translation region

As a result, material transportation underlying the extrusion course was a combination of two transportation, one was positive displacement pumping transportation in the intermeshing region, and the other was partially filled transportation in the translation region。

\section{VIRTUAL ASSEMBLY ${ }^{[9]}$}

Virtual assembly is a kind of technique to take into the comprehensive usage of $\mathrm{CAD}$ technique, visualization technique, and simulated technique. Assembly of virtual prototype is worked out in the virtual environment without the real physical model and real process of assembly. 


\subsection{Process of virtual assembly}

In the process of virtual assembly, assembly plan must be carried out first, it includes the enough information to support the process choice decision, and acquire the more detailed process definition, consider the relationship of hierarchy and constraint between parts and subassemblies, estimate the feasibility of assembly, choose the good assembling sequence and path. At the same time, the moving path of each parts need to be recorded in the assembling space environment, thus these paths become the soft districts, need to carry out the method to establish, save and review these soft districts.

The assembling description in virtual environment is reflected to the real assembling process. Virtual assembly will produce some useful information, e.g. assembly interference, sequence and path etc. and the information should be sent to the CAD system to modify the $3 \mathrm{D}$ model further. fig. 4 is the process of virtual assembly.

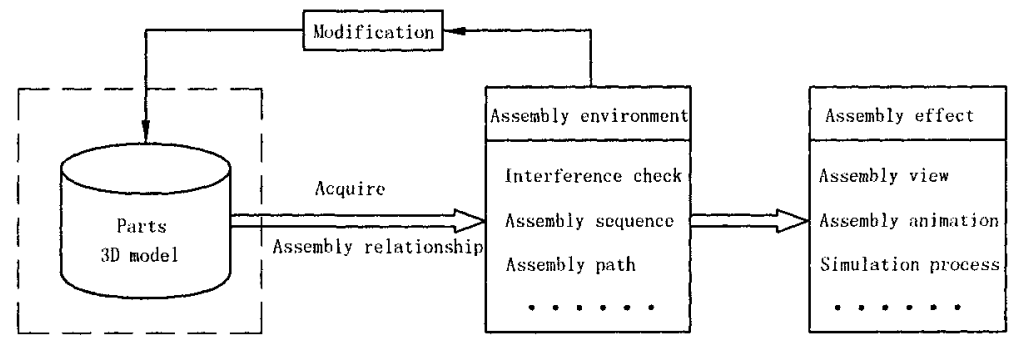

Figure 4. Process of virtual assembly

\section{2 Interference check in virtual assembly environment}

Taking Teamcenter Visualization software as the virtual environment, rice bran extruder is designed with $3 \mathrm{D}$ software, then these $3 \mathrm{D}$ model is transferred into the Teamcenter Visualization to assemble visually.

Interference check needs to set the check scope first, you can select the shown parts on the screen or all parts of prototype. Moreover clearance needs to setup, and run the interference check program, environment will show you the interference between every two parts which have been assembled each other, and the report is in a matrix list. Such as the fig.5 show, the diagram is the interference circumstance of the mould and the barrel head, the manifestation of interference is penetration, this is 
highlighted with the red color on the entity, and the section view shows the section circumstance clearly. This interference can be removed by the further modification.

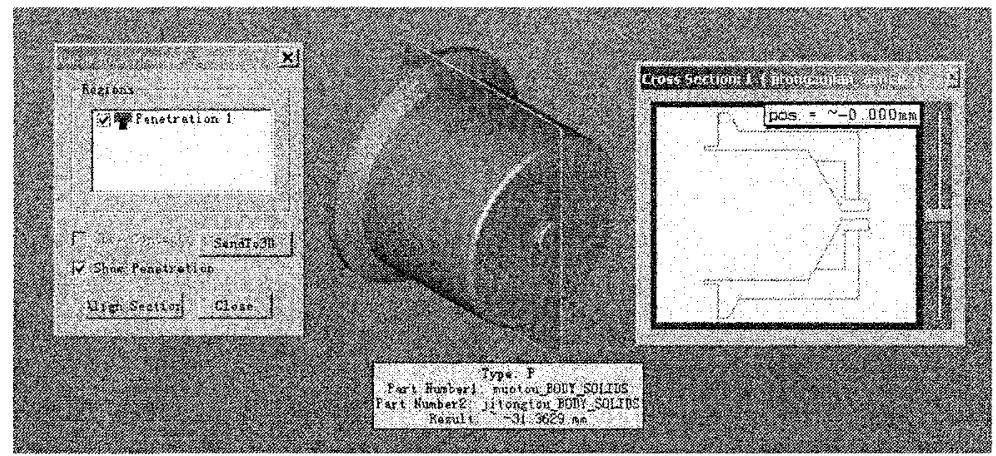

Figure 5. Interference check in virtual assembly environment

\section{3 Virtual assembly sequence and path}

To create the assembly sequence and path should disassemble the assembly model artificially, base on the supposition which assembly is disassembly available, we can acquire the sequence and path by tracking parts reversely. Such as fig. 6 show, the diagram is the assembly sequence and path of screw subassembly, small balls indicate the assembly paths, the sequence of these paths also means the assembly sequence.

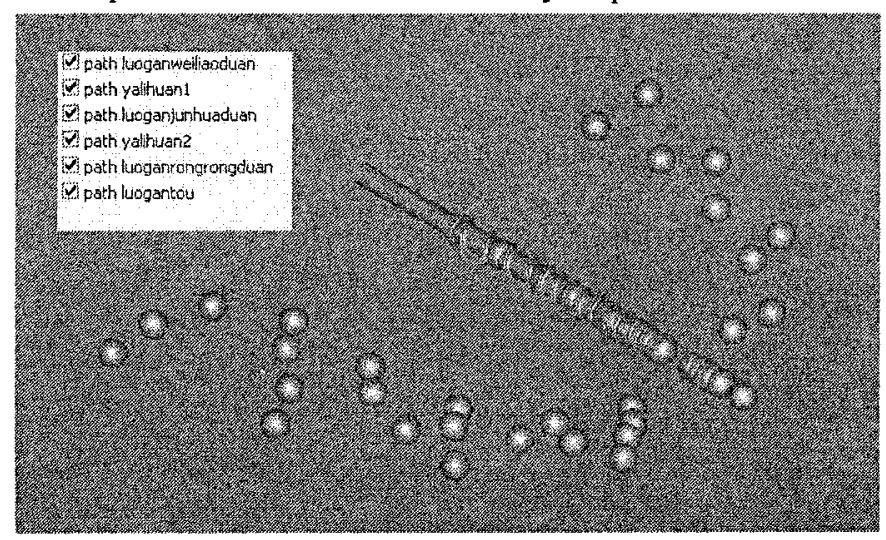

Figure 6. Assembly sequence and path layout

Assembly animation can be established to demonstrate an assembly sequence or some other series actions, firstly we specify the animation properties to control the whole speed of animation and keep on time, then 
add a series of events, these events include assembly path, and Organize actions into events, thus broadcast the animation to validate the assembly process, further edit the animation and create the movie document of AVI. Finally the animation of virtual assembly is completed.

\section{CONCLUSION}

Visualization technique is modern mean to research the complicated problem of engineering. We make use of visualization technique to open the "Black Box" that it is hard to understand at former time.

Visual experiments has been done on the process of rice bran extrusion, the phenomena indicate that material transportation underlying the extrusion course is a combination of two transportation, one is positive displacement pumping transportation in the intermeshing region, and the other is partially filled transportation in the translation region; extrusion of rice bran is a process of cooking at high temperature and under high pressure 。

Virtual assembly technique has been used to design the extrusion assembly, and interference is checked, and animations are created to demonstrate assembly sequence and path. This product has been designed successfully by computer in virtual environment.

\section{REFERENCES}

1. Connor, Colm, (1987), Extrusion Technology For the Food Industry, Great Britain: Galliard Ltd, Great Yarmouth.

2. Loeb, J.R., et al., (1949), Storage of the Bran as It Affects Hydrolysis of the Oil, Journal of the American Oil Chemists' Society, vol.27, pp738 743.

3. Williams, M., and Baer, S., (1965), The expansion and extraction of rice bran, Journal of the American Oil Chemists' Society, vol.42, pp151 155.

4. Prabhakar, J.V., et al., (1986), A Simple Chemical Method for Stabilization of Rice Bran, Journal of the American Oil Chemists 'Society, vol.63(5), pp644 648.

5. Tao, J., et al., (1993), Microwave Heating for Rice Bran Stabilization, Journal of Microwave Power Electromagnetic Energy, vol.28(3), pp156 159.

6. Fernando, W.J.N., et al., (1993), Effect of Fluidized Bed Drying on Stabilization of Rice Bran, Drying Technology, vol.11(5), ppl115 1119.

7. The RiceX Company--Feeding the World, (2000), [EB/OL] www.RiceX.com

8. Zhu Wen-hua, (2001), Studies on Stabilization of Rice bran by Extrusion Cooking, PhD. Dissertation of Southern Yangtze University, (in Chinese).

9. Zhu Wen-hua, Ma Deng-zhe, (2004). Application Research of Virtual Assembly Technology, Journal of Design and Research, vol.94(6), pp47 48, (in Chinese). 